'Instituto Oncológico, Viña del Mar, Universidad de Valparaíso, Chile.

2IRAM, Santiago, Chile. ${ }^{3}$ Instituto Nacional del Cáncer Santiago, Chile.

${ }^{4}$ Clínica Alemana, INTOP, Santiago, Chile.

'Clínica Alemana, Instituto Nacional Del Cáncer, Santiago, Chile. ${ }^{6}$ Pontificia Universidad Católica de Chile, Santiago. Chile. 'Hospital Regional de Concepción Chile.

${ }^{8}$ Radionuclear, Santiago, Chile ${ }^{9}$ Clínica Santa María, Santiago, Chile.

${ }^{10}$ Fundación Arturo López Pérez, Santiago, Chile

Recibido el 26 de marzo de 2012, aceptado el 28 de mayo de 2012 .

Correspondencia a:

Dr. Pablo González Mella Instituto Oncológico. Hospital Naval Almirante NefClínica Reñaca.

Departamento de Oncología. Universidad de Valparaíso. Anabaena 336, Jardín del Mar, Reñaca, Viña del Mar, Chile. Fono: 56-32-2830516. E-mail:pagon@ institutooncologico.cl

\section{Terapias perioperatorias en el cáncer de vesícula biliar resecable: conclusiones del Consenso Latinoamericano de Manejo del Cáncer de Vesícula Biliar}

\author{
PABLO GONZÁLEZ M. ${ }^{1}$, RAMÓN BAEZA ${ }^{2}$, BETTINA MÜLLER $^{3}$, \\ JORGE GALLARDO ${ }^{4}$, HANS HARBST ${ }^{5}$, MARISA BUSTOS ${ }^{6}$, \\ CÉSAR GARCÍA ${ }^{7}$, OSVALDO GIANNINI ${ }^{1}$, RAÚL CARTES ${ }^{8}$, \\ EUGENIO VINÉS ${ }^{9}$, PAULA CARRASCO ${ }^{10}$
}

\section{Radio and chemotherapy in gallbladder cancer. A Latin American Consensus}

\begin{abstract}
No randomized controlled trials have been conducted in gallbladder cancer to establish standard treatments. We therefore conducted the first Latin American Consensus meeting for the management of gallbladder cancer. In this paper we report the conclusions of the experts' panel for (neo) adjuvant treatment of resectable gallbladder cancer. These are based on the review of the literature, the discussion of the participating experts and the vote of the assistants (surgical oncologists, medical oncologists, radiation oncologists and others). The reviewed topics were the role or adjuvant radiochemotherapy in T1bNOMO, T2-3 NO-1 MO and T4 NO-1 MO disease and doses, schedules and drugs for radiochemotherapy.

(Rev Med Chile 2012; 140: 1060-1066).

Key words: Adjuvants, immunological; Chemoradio therapy; Drug therapy); Gallbladder neoplasms; Radiotherapy.
\end{abstract}

$\mathrm{E}$ 1 cáncer de vesícula biliar es una enfermedad muy poco frecuente en Europa y Norteamérica, con una incidencia de 1,2 por 100.000 habitantes $^{1}$, y con escaso e interés científico; sin embargo, es de alto impacto en áreas de Sudamérica como en Chile, donde constituye la primera causa de muerte por cáncer en mujeres. Particularmente la región de la Araucanía presenta la mayor tasa de mortalidad reportada en el mundo (35 por 100.000 habitantes/año). Otras regiones con una alta incidencia del cáncer de vesícula biliar son Bolivia, Perú, Ecuador, Colombia, Brasil, la población amerindia de Estados Unidos de Norteamérica, Polonia, Argelia, India ${ }^{2}$ y el norte andino de Argentina.

El pronóstico del cáncer de vesícula biliar sigue siendo ominoso, con una sobrevida global de sólo
$5 \%$ a 5 años ${ }^{3}$. Incluso en pacientes resecados con cirugía de intención curativa en estadios tempranas (T2N0M0), la supervivencia a 5 años no llega al $30 \%{ }^{4,5}$, lo cual se explica por un alto porcentaje de pacientes que presentará recurrencias tanto locoregionales como a distancia. Muchos pacientes son diagnosticados en etapas avanzadas, fuera del alcance quirúrgico.

En el contexto del Simposio Latinoamericano de Gastroenterología Oncológica SLAGO 2009, celebrado en la ciudad de Viña del Mar, se desarrolló el primer Consenso Latinoamericano de Cáncer de Vesícula, que abordó los temas de manejo quirúrgico, terapias perioperatorias y terapias paliativas. En la presente publicación presentamos las conclusiones referentes a las terapias perioperatorias en pacientes con enfermedad resecable, basadas 
en las presentaciones y votaciones vertidas en el simposio por la audiencia, representada en un 33\% por cirujanos oncólogos, en $24 \%$ por oncólogos médicos, en $20 \%$ por oncólogos radioterapeutas y en $23 \%$ por profesionales vinculados a la oncología (enfermeras especializadas en oncología, químicos farmacéuticos, psico-oncólogos y algunos residentes en especialidades oncológicas invitados), además de la revisión exhaustiva de la literatura y la discusión del panel de expertos. En la audiencia y como expertos votantes se encontraban especialistas destacados de Chile, Argentina, Uruguay, Ecuador, Bolivia, Paraguay, Perú, México, Venezuela, Brasil, Panamá, Guatemala y Estados Unidos de Norteamérica. Las conclusiones referentes a las terapias paliativas fueron recientemente reportadas en esta misma revista ${ }^{6}$.

\section{Conclusiones del Consenso}

\section{Pacientes T1b NO MO (infiltrantes hasta la muscular, AJCC $6^{a}$ Edición)}

\section{1. ¿ Existe indicación de radio-quimioterapia adyu-} vante en pacientes sometidos a colecistectomía simple?

Existe consenso en la literatura que en la enfermedad T1a (limitada a la mucosa) el riesgo de compromiso ganglionar es inferior a 3\% y el riesgo de recidiva extremadamente bajo por lo que no se justifica someter al paciente a una cirugía radical ni a radio-quimioterapia adyuvante. La situación es más compleja en la enfermedad T1bN0M0. Esta situación clínica fue el primer punto a revisar y consensuar.

Se realizó una revisión del riesgo de compromiso ganglionar, metástasis a distancia y cifras de sobrevida global. El riesgo de compromiso ganglionar es cercano al 15\% ,y 50 de los pacientes presenta elementos histológicos que podrían conferir un alto riesgo (permeación vascular linfática y/o perineural). Las cifras de mortalidad a 5 años, aún luego de cirugía radical son cercanas a $15 \%^{7-10}$. Los expertos revisores indican que este grupo amerita el ingreso a estudios clínicos para evaluar el rol de la cirugía radical y/o radioquimioterapia complementaria. Fuera de protocolos de investigación, los expertos votantes no recomiendan, en forma mayoritaria, la utilización de terapias complementarias.
El Consenso recomienda la inclusión de este grupo a protocolos de investigación, fuera de ellos recomienda la observación en pacientes portadores de enfermedad T1b N0 M0 sometidos a simple colecistectomía.

2. ¿ Existe indicación de radio-quimioterapia adyuvante luego de colecistectomía radical?.

Se reconoce el rol etapificador de una cirugía radical pero se desconoce su impacto terapéutico. Los expertos votantes no recomiendan, en forma mayoritaria, la utilización de terapias complementarias.

El Consenso recomienda la inclusión de estos pacientes a protocolos de investigación, fuera de ellos recomienda la observación en pacientes portadores de enfermedad T1b N0 M0, sometidos a colecistectomía radical.

\section{Pacientes T2 -3 N0-1 M0}

3. ¿Existe indicación de radio-quimioterapia adyuvante en todos los pacientes?

Si se analiza las sobrevidas a 5 años de pacientes con compromiso de la subserosa y/o de la serosa sometidos a cirugía exclusiva nos encontramos con cifras de sobrevida de 37 y $15 \%$ respectivamente en la serie con mayor número de pacientes ${ }^{7}$. La mayor serie reportada en Chile demuestra resultados equivalentes ${ }^{11}$. Cuando se describe el patrón de recidiva de los pacientes resecados, el $80 \%$ presenta un componente loco-regional lo que ofrece una base racional para terapias adyuvantes regionales ${ }^{12}$. Un estudio retrospectivo con sobre 4.000 pacientes reportó mediante un análisis multivariado que la radioterapia postoperatoria produce beneficio en sobrevida a los pacientes con enfermedad más avanzada o igual a $\mathrm{T}^{13}$. Los resultados de series reportadas con radioquimioterapia adyuvante son superiores a los descritos con cirugía exclusiva, y la tolerancia razonablemente buena ${ }^{13-18}$. Los expertos votantes, en forma mayoritaria recomienda radioquimioterapia postoperatoria a este grupo de pacientes.

El Consenso reconoce la falta de estudios aleatorios y sugiere fuertemente la realización de estos para obtener conclusiones más definitivas. La mejor evidencia disponible sin embargo, permite recomendar la radioquimioterapia adyuvante en atención al patrón de recidiva y la existencia de series que muestran una tolerancia aceptable y resultados superiores a los reportados con cirugía exclusiva. 
4. Los hallazgos en la pieza operatoria luego de la reintervención radical, ¿determinan la indicación de terapia adyuvante?

Existen diversos factores pronósticos de recidiva y de sobrevida que sólo pueden identificarse en los hallazgos de la pieza operatoria definitiva. Entre ellos el tipo de resección, R0 versus R1, demostrándose la inexistencia de sobrevivientes a largo plazo cuando existe resección con enfermedad residual macroscópica o microscópica, el grado de invasión parietal, T1b versus T2 versus T3. N0 versus N1,la presencia de invasión linfovascular, grado de diferenciación y el subtipo papilar ${ }^{13}$. Todos ellos deben ser considerados en el momento de evaluar el riesgo de recidiva; sin embargo, no se ha identificado en forma prospectiva el beneficio de una radioquimioterapia adyuvante en la presencia de estos factores. Los expertos revisores y la mayoría de los votantes consideran estos factores al momento de decidir una adyuvancia luego de una cirugía radical, particularmente en la enfermedad precoz T1b N0 M0, pero no modifican la conducta pro tratamiento adyuvante en la enfermedad T2-3 N0-1 M0 ${ }^{13-15}$.

El Consenso reconoce la presencia de factores pronósticos en la pieza operatoria de la cirugía radical y la ausencia, sin embargo, de factores predictivos del beneficio de la radioquimioterapia adyuvante. Se reconoce a los pacientes R1-2 y M+ (incluyendo ganglios lumboaórticos que corresponden a N2 en AJCC, versión 7a) como candidatos a manejo exclusivamente paliativo. Los otros factores no son, en la actualidad, gravitantes en la decisión de realizar o no una radioquimioterapia adyuvante en la enfermedad T2-3 N0-1 M0.

\section{5. ¿Existe algún rol de terapias neoadyuvantes?}

Luego de una colecistectomía con hallazgo de un cáncer de vesícula biliar T2-3 NX M0, existe consenso en la necesidad de realizar una reoperación radical por su rol etapificador y eventualmente terapéutico (no demostrado aún en estudios randomizados fase III). Desafortunadamente es frecuente que se obtenga una resección R1 R2, con un mal pronóstico a corto plazo. Parece racional plantear una terapia neoadyuvante, previa a la reoperación radical con el objetivo de incrementar las cifras de resección R0, único procedimiento potencialmente curativo.

Hemos identificado un solo estudio no rando- mizado, chileno, que evaluó la radioquimioterapia neoadyuvante en cáncer de vesícula biliar potencialmente resecables, luego de una colecistectomía. Durante el consenso se revisaron los resultados con una mediana de seguimiento de 62 meses, en 21 pacientes T2-3, N0-1. Se reportó $87 \%$ de resecciones R0 y una sobrevida a 5 años de $57 \%$, superior a los resultados históricos en el mismo centro ${ }^{19,20}$. No queda claro los criterios de inclusión, el tipo de estudio (retrospectivo o prospectivo), el número de pacientes a ingresar y el objetivo primario del estudio.

El Consenso de los revisores y la mayoría de los expertos votantes concluyen que no existe a la fecha evidencia suficiente para recomendar terapias neoadyuvantes en el cáncer de vesícula biliar fuera de un estudio de investigación.

\section{Pacientes T4 NO-1 MO}

6. ¿Son candidatos a radio-quimioterapia adyuvante con intención curativa?

Los pacientes con enfermedad T4 independientemente de su status ganglionar presentan enfermedad irresecable en condiciones habituales por su compromiso vascular o compromiso de dos o más órganos extrahepáticos (AJCC versión $6^{\mathrm{a}}$ y $7^{\mathrm{a}}$ ). En circunstancias muy particulares podría ser resecado un paciente en estas condiciones sin embargo, esta debe considerarse al menos R1. Se revisa la literatura, las opiniones de expertos y no se encuentra evidencias de un beneficio real de una radioquimioterapia adyuvante. La sala mayoritariamente vota en contra de la realización de radioquimioterapia adyuvante en este contexto.

El Consenso concluye no recomendar la realización de radioquimioterapia adyuvante en la enfermedad T4.

\section{7. ¿Existe algún rol de terapias neoadyuvantes?}

Se reconoce la necesidad de contar con terapias activas capaces de conseguir un "downstaging" debido a la alta frecuencia con la que se diagnostica esta enfermedad en fase avanzada, no resecable con intención curativa.

Se revisa el único estudio publicado con radioquimioterapia neoadyuvante. No fueron tratados pacientes $\mathrm{T} 4$, la técnica de radioquimioterapia es obsoleta y el número de pacientes escaso ${ }^{19,20}$. Se reúnen las opiniones de expertos y las votaciones de la sala. 
El Consenso concluye que no hay datos que justifiquen, fuera de un protocolo de investigación, la realización de radioquimioterapia neoadyuvante en enfermedad T4.

\section{Radio-quimioterapia:}

8. Radioterapia con 5 FU, capecitabina o gemcitabina concomitante. Dosis esquemas.

La asociación de radioterapia y quimioterapia concomitante utilizada a título adyuvante o neoadyuvante ha representado el principal avance en el tratamiento de distintos cánceres digestivos. Se ha demostrado beneficios en sobrevida global en tumores de la vía aéreo-digestiva superior, esófago, gástrico, páncreas y recto ${ }^{21-27}$. Se ha reportado beneficio en control locorregional y calidad de vida, con su utilización a título exclusivo, en el cáncer de canal anal' ${ }^{28}$.

Lo anterior hace planteable su utilización en el cáncer de la vesícula biliar. La mejor evidencia disponible se basa exclusivamente en series retrospectivas o prospectivas no randomizados con un bajo número de pacientes. La mayor experiencia se tiene con radioterapia de alta energía, con fraccionamientos convencionales, de 1.8 a 2 Gy por fracción, 5 fracciones por semana con dosis total de 45 a 54 Gy. La quimioterapia concomitante más utilizada es con $5 \mathrm{FU}$ en bolus semana 1 y 5 , infusión continua durante todo el tratamiento o capecitabina durante todo el tratamiento. Los expertos revisores recomiendan mayoritariamente, a falta de evidencia en vesícula biliar, utilizar esquemas validados en recto. La gemcitabina se ha utilizado en un reducido número de pacientes, se reconoce su potente efecto radiosensibilizante y su mayor morbilidad potencial al compararla con las flouropirimidinas. No hay claridad respecto de la dosis adecuada, en general se ha extrapolado los esquemas utilizados en páncreas, los que a su vez no están del todo consensuados ${ }^{29-32}$.

Se revisa la literatura, y fuera de protocolos de investigación, se recomienda la utilización de flouropirimidinas en forma mantenida durante la radioterapia. Por razones de comodidad, preferencia de los pacientes y de orden práctico, la audiencia de expertos votantes recomienda como primera alternativa la capecitabina en dosis de $825 \mathrm{mgr} / \mathrm{m}^{2}$ repartido en dos dosis al día. En segundo orden el 5-FU en infusión continua en dosis de $225 \mathrm{mg} /$ $\mathrm{m}^{2}$ al día. Finalmente, sólo 15\% de la audiencia recomienda la gemcitabina concomitante, sin embargo, los estudios fase I para determinar su dosis ideal concomitante con radioterapia (en el cáncer de páncreas) varían entre $60 \mathrm{mg} / \mathrm{m}^{2}$ por dos veces a la semana, hasta $1.000 \mathrm{mgr} / \mathrm{m}^{2}$ una vez por semana ${ }^{29-32}$.

El Consenso recomienda la utilización de radioterapia con fraccionamiento convencional, en dosis de 45 Gy con sobreimpresión hasta 50,4 Gy con campos reducidos. Se recomienda la utilización de flouropirimidinas en forma concomitante.

9. ¿Existe algún rol de la quimioterapia (neo) adyuvante exclusiva?. Dosis esquemas

No se ha publicado hasta la fecha un estudio aleatorio de quimioterapia adyuvante en cáncer de vesícula biliar, por lo que no se ha podido establecer un tratamiento estándar ampliamente aceptado. En un estudio japonés que incluyó pacientes con carcinoma pancreáticobiliar, y que evaluó un total de 112 pacientes con cáncer de vesícula biliar, se reportó un beneficio en sobrevida global con quimioterapia adyuvante con mitomicina $\mathrm{C}+$ 5 -fluorouracilo (sobrevida a 5 años de $26 \%$ versus $14,4 \%, \mathrm{p}=0,0357, \mathrm{HR}=0,654^{33}$. Recientemente se reportaron dos estudios randomizados que demostraron un impacto favorable en sobrevida global con esquemas de quimioterapia de gemcitabina y gemcitabina asociada con cisplatino a título paliativo, lo cual hace urgente explorar este tipo de esquemas a título adyuvante en estudios randomizados controlados con un diseño y poder estadístico adecuado ${ }^{34,35}$.

El consenso recomienda la conducción de un estudio randomizado que explore el rol de la quimioterapia adyuvante en el cáncer de vesícula biliar resecado en forma curativa. No se recomienda el uso de quimioterapia adyuvante exclusiva fuera de estudios clínicos.

\section{0. ¿Cuáles son los requerimientos técnicos mínimos} para una correcta radioterapia en el cáncer de vesícula biliar?. Campos, CTV, PTV, Energías, técnica convencional vs conformacional 3D vs IMRT vs IGRT.

Se reconoce la radioterapia como una herramienta de enorme impacto en los cánceres en general y de los digestivos en particular. Existe numerosas evidencias que demuestran que el beneficio obtenido por el efecto antitumoral de 
Tabla 1. Restricciones órgano / volumen blanco

\begin{tabular}{|lcl|}
\hline $\begin{array}{l}\text { Órganos de riesgo/ } \\
\text { Volumen blanco }\end{array}$ & Volumen & Restricción \\
\hline PTV & $100 \%$ & 50.4 Gy mínimo \\
\hline Intestino delgado & $100 \%$ & 45 Gy máximo \\
& $75 \%$ & 48 Gy máximo \\
& $50 \%$ & 50 Gy máximo \\
& $25 \%$ & 55 Gy máximo \\
Riñón derecho & $50 \%$ & 18 Gy máximo \\
& $25 \%$ & 20 Gy máximo \\
Riñón izquierdo & $50 \%$ & 10 Gy máximo \\
& $25 \%$ & 15 Gy máximo \\
Hígado & $50 \%$ & 30 Gy máximo \\
Médula espinal $+0,5 \mathrm{~cm}$ & $100 \%$ & 45 Gy máximo \\
\hline
\end{tabular}

la radioterapia puede perderse e incluso tener un efecto deletéreo si no se realiza un control de calidad y no respetan las condiciones técnicas necesarias $^{36-39}$.

Se revisan los requerimientos mínimos necesarios para realizar una adecuada radioterapia en el cáncer de la vesícula biliar.

La realización de simulación con TAC que permita obtener imágenes de reconstrucción $3 \mathrm{D}$ y en particular la obtención de BEV (beam eye view) y $D R R$ (digitally reconstructed radiography) junto con dosimetría 3D, constituye un requerimiento técnico mínimo de la Radioterapia Conformacional 3D.

A la fecha no existe una clara evidencia del beneficio en términos de sobrevida con radioterapia con modulación de intensidad (IMRT, procedimiento técnico que es posible gracias a la incorporación de un software de planificación inversa cuya principal ventaja es la posibilidad de generar curvas de isodosis cóncavas) ni con radioterapia guiada por imágenes (IGRT, procedimiento que permite además tener en cuenta la movilidad de los órganos y volúmenes blanco, gracias a que incorpora equipos de imaginería montados en el equipo de radioterapia, de manera de realizar correcciones del posicionamiento en tiempo real), sin embargo, su potencial beneficio en términos de disminuir la toxicidad aguda y crónica es algo que debe ser estudiado y se estimula la generación de estudios en este sentido.
Los expertos revisores y los especialistas votantes concluyen que la realización de radioterapia conformacional $3 \mathrm{D}$, con múltiples campos (al menos 3), y un estricto respeto de los OAR (organ at risk) sin sacrificar la dosis al PTV (planning tumor volumen) constituyen una exigencia absoluta al momento de plantear una radioquimioterapia adyuvante. Se recomiendan las siguientes restricciones (Tabla 1).

El Consenso destaca la necesidad contar con un programa de control de calidad de la radioterapia. Se reconoce como un mínimo necesario la utilización de radioterapia conformacional $3 \mathrm{D}$ con múltiples campos.

\section{Referencias}

1. Carriaga M, Henson D. Liver, gallbladder, extrahepatic bile ducts, and pancreas. Cancer 1995; 75 (1 Suppl): 17190.

2. Lazcano-Ponce E, Miquel J, Muñoz N, Herrero R, Ferrecio C, Wistuba I, et al. Epidemiology and Molecular Pathology of Gallbladder Cancer. CA Cancer J Clin 2001; 51: 349-64.

3. Cubertafond P, Gianant A, Cucchiaro G. Surgical treatment of 724 carcinomas of the gallbladder: Results of the French Surgical Association Survey. Ann Surg 1994; 219 (3): 275-80.

4. Greene F, Page D, Fleming I, Fritz A, Balch CM, Haller DG, Morrow M. AJCC Cancer Staging Manual, 6th ed. 2002; New York: Springer.

5. Fong Y, Wagman L, Gonen M, Crawford J, Reed W, Swanson R, et al. Evidence-Based Gallbladder Cancer Staging-Changing Cancer Staging by Analysis of Data From the National Cancer Database. Ann Surg 2006; 243 (6): 767-74.

6. Müller B, Arroyo G, Gallardo J, Villanueva L, González $\mathrm{P}$, Baeza R, et al. Manejo paliativo del cáncer de vesícula biliar irresecable o metastásico: Conclusiones del Consenso Latinoamericano de Manejo del Cáncer de Vesícula Biliar. Rev Med Chile 2011; 137 (2).

7. Ogura Y, Mizumoto R, Isaji S, Kusuda T, Matsuda S, Tabata M. Radical operations for carcinoma of the gallbladder. Present status in Japan. World J Surg 1991; 15: 337-43.

8. Varshney S, Buttirini G, Gupta R. Incidental carcinoma of the gallbladder. Eur J Surg Oncol 2002; 28: 4-10.

9. Yuldirim E, Celen O, Gulben K, Berbereglu U. The surgical management of incidental gallbladder carcinoma. Eur J Surg Oncol 2005; 31: 45-52. 
10. Wakai T, Shirai Y, Yokohama N, Nagakura S, Watabe $\mathrm{H}$, Hatakeyama. Early gallbladder carcinoma does not warrat radical resection. Br J Surg 2001; 88: 675-8.

11. Roa I, Araya J, Villaseca M, Roa J, Ibacache G, Burgos L, Muñoz S. Elementos morfológicos pronósticos en el cáncer de la vesícula biliar. Rev Med Chile 2002; 130: 387-395.

12. Bosset J, Mantion G, Gillet M, Pelissier E, Boulanger M, Maingon P, et al. Primary carcinoma of the gallbladder. Adjuvant postoperative external irradiation. Cancer 1989; 64: 1843-7.

13. Wang S, Fuller C, Kim J, Sittig D, Thomas C, Ravdin P. Prediction Model for Estimating the Survival Benefit of Adjuvant Radiotherapy for Gallbladder Cancer. J Clin Oncol 2008; 26: 2112-7.

14. Baeza M, Reyes J, Del Castillo C, Rivera R. Post-operative adjuvant radio-chemotherapy in the treatment of gallbladder cancer. Int J Radiat Oncol Biol Phys 2005; 63 (Sup 1): s285-6.

15. Czito BC, Hurwitz H, Clough R, Tyler D, Morse M, Clary B, et al. Adjuvant external beam radiotherapy with concurrent chemotherapy after resection of primary gallbladder carcinoma: a 23 year experience. Int J Radiat Oncol Biol Phys 2005; 62: 1030-4.

16. Kresl J, Schild S, Henning G, Gunderson L, Donohue J, Pitot $\mathrm{H}$, et al. Adyuvant external beam radiation therapy with concurrent chemotherapy in the management of gallbladder carcinoma. Int J Radiat Oncol Biol Phys 2002; 52 (1): 167-75.

17. Gold J, Miller R, Haddock M, Gunderson L, Quevedo F, Donohue J, et al. Adjuvant therapy for gallbladder carcinoma. The Mayo Clinic Experience. Int J Radiat Oncol Biol Phys 2009; 75: 150-5.

18. Pahlajani N, Ruth K, Freedman G, Meropol M, Cohen S, Watson J, et al. Radiation therapy in gallbladder cancer treatment: The Fox Chase Cancer Center Experience [Abstract]. Int J Radiat Oncol Biol Phys 2007; 69 (Suppl. 1): $S 301$.

19. De Aretxabala X, Roa I, Burgos L, Cartes F, Silva J, Yáñez E, et al. Preoperative chemoradiotherapy in the treatment of gallbladder cancer. Am Surg 1999; 65: 2416.

20. De Aretxabala, Losada H, Mora J, Roa I, Burgos L, Yáñez E, et al. Quimiorradioterapia de neoadyuvancia en cáncer de vesícula biliar. Rev Med Chile 2004; 132: 51-7.

21. Pignon J, le Maitre A, Bourhis J. Meta-analyses of chemotherapy in head and neck cancer (MACH-NC): An update. Int J Radiat Oncol Biol Phys 2007; 69: S112-4.

22.- Al-Sarraf M, Leblanc M, Giri S, Fu K, Cooper J, Vuong $\mathrm{T}$, et al. Chemoradiotherapy versus radiotherapy in patients with advanced nasopharyngeal cancer: Phase III randomized Intergroup Study 0099. J Clin Oncol 1998; 16: 1310-7.

23. Herskovic A, Martz K, al-Sarraf M, Leichman L, Brindle J, Vaitkevicius V, et al. Combined chemotherapy and radiotherapy compared with radiotherapy alone in patients with cancer of the esophagus. N Engl J Med 1992; 326: 1593-8.

24. Walsh T, Noonan N, Hollywood D, Kelly A, Keeling, Hennessy T. A comparison of multimodal therapy and surgery for esophageal adenocarcinoma. N Engl J Med 1996; 335: 462-7.

25. Macdonald J, Smalley S, Benedetti J, Hundahal S, Estes $\mathrm{N}$, Stemmermann G, Haller D, et al. Chemoradiotherapy after surgery compared with surgery alone for adenocarcinoma of the stomach or gastroesophageal junction. $\mathrm{N}$ Engl J Med 2001; 345: 725-30.

26. Klinkenbijl J, Jeekel J, Sahmaud T, van Pel R, Couvreur $\mathrm{M}$ Veenhof C, et al. Adjuvant radiotherapy and 5-flourouracil after curative resection of cancer of the pancreas and periampullary region: phase III trial of the EORTC gastrointestinal cancer cooperative group. Ann Surg 1999; 230 (6): 776-82.

27. Krook J, Moertel C, Gunderson L, Wieand H, Collins R, Collins R, et al. Effective surgical adjuvant therapy for high risk rectal carcinoma. N Engl J Med 1991; 324 (11): 709-15.

28. Bartelink H, Roelofsen F, Eschwège F, Rougier, Bosset J, González D, et al. Concomitant radiotherapy and chemotherapy is superior to radiotherapy alone in the treatment of locally advanced anal cancer (results of a phase III randomized trial of the European Organization for Research and Treatment of Cancer Radiotherapy and Gastrointestinal Cooperative Groups). J Clin Oncol 1997; 15: 2040-204.

29. Small W, Berlin J, Freedman G, Lawrence T, Talamonte M, Mulcahy M, et al. Full Dose Gemcitabine With Concurrent Radiation Therapy in Patients with Non Metastatic Pancreatic Cancer. A Multicenter Phase II Trial. J Clin Oncol 2008; 26: 942-947.

30. Girard N, Mornex F, Bossard N, Ychou M, Chauffert B, Wautot V. Estimating Optimal Dose of Twice-Weekly Gemcitabine for Concurrent Chemoradiotherapy in Unresectable Pancreatic Carcinoma: Mature Results of GEMRT-01 Phase I Trial. Int J Radiat Oncol Biol Phys 2010; 77 (5): 1426-32.

31. Blackstock W, Bernard S, Richards F, Eagle K, Case D, Poole M, et al. Phase I Trial of Twice-Weekly Gemcitabine and Concurrent Radiation in Patients With Advanced Pancreatic Cancer. J Clin Oncol 1999; 17: 2208-12. 
32. McGinn C, Zalupski M, Shureiqi I, Robertson J, Eckhauser E, Smith D, et al. Phase I Trial of Radiation Dose Escalation With Concurrent Weekly Full-Dose Gemcitabine in Patients With Advanced Pancreatic Cancer. J Clin Oncol 2001; 19: 4202-8.

33. Takada T, Amano H, Yasuda H, Nimura Y, Matsushiro T, Nagakahua T, et al. Is postoperative adjuvant chemotherapy useful for gallbladder carcinoma? A phase III multicenter prospective randomized controlled trial in patients with resected pancreaticobiliary carcinoma, Cancer 2002; 95 (8): 1685-95.

34. Valle J, Wasah H, Palmer D, Cunningham D, Anthoney A, Maraveyas A, et al. Cisplatin plus Gemcitabine versus Gemcitabine for Biliary Tract Cancer. N Engl J Med 2010; 362 (14): 1273-81.

35. Sharma A, Dwary A D, Mohanti B K, Deo S V, Pal S, Sreenivas V, et al. Best Supprotive Care Compared UIT Chemotherapy for Unresectable Gall Bladder Cancer: A
Randomized Controlled Study. J Clin Oncol 2010; 28: 4581-6.

36. Cuzick J, Steward H, Peto R, Fisher B, Kaae S, Johansen $\mathrm{H}$, et al. Overview of Randomized trials of Postoperative Adjuvant Radiotherapy in Breast Cancer. Cancer Treat Rep 1987; 71: 15-29.

37. Cuzick J, Stewart H, Rutqvist L, Houghton J, Edwards R, Redmond C, et al. Cause specific mortality in long term survivors of breast cancer who participated in trials of radiotherapy. J Clin Oncol 1994; 12: 447-5.

38. PORT Meta-analysis Trialists Group. Postoperative radiotherapy in non small cell lung cancer; systematic review and meta-analysis of individual patient data from ninerandomized controlled trials. Lancet 1998; 352: 257-63.

39. Neoptolemos J, Stocken D, Fries H, Bassi C, Dunn J, Hickey H, et al. A Randomized Trial of Chemoradiotherapy and Chemotherapy after Resection of Pancreatic Cancer. N Engl J Med 2004; 350: 1200-10. 